\title{
Self-Powered Sensor for Quantifying Ocean Surface Water Waves Based on Triboelectric Nanogenerator
}

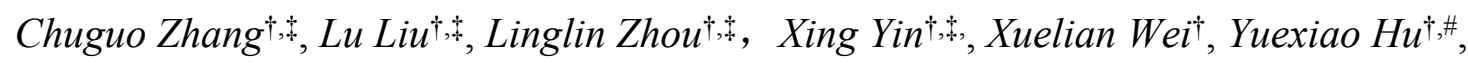

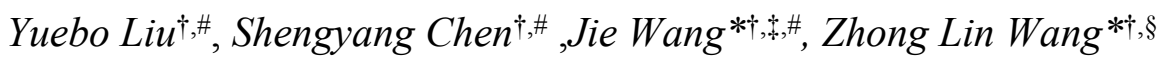

${ }^{\dagger}$ Beijing Institute of Nanoenergy and Nanosystems, Chinese Academy of Sciences, Beijing 100083, P. R. China

College of Nanoscience and Technology, University of Chinese Academy of Sciences, Beijing 100049, P. R. China

\$School of Materials Science and Engineering, Georgia Institute of Technology, Atlanta, GA 30332, USA

${ }^{\#}$ Center on Nanoenergy Research, School of Physical Science and Technology, Guangxi University, Nanning, 530004, P. R. China

\section{Corresponding Authors}

*Email: wangjie@binn.cas.cn

*Email: zhong.wang@mse.gatech.edu

\section{Author Contributions}

Chuguo Zhang, Lu Liu, Linglin Zhou contributed equally to this work. 


\section{Supplementary Notes}

\section{Supplementary Note1 :}

When the buoy drives the TOSS, friction between PTFE and $\mathrm{Cu}$ electrode will be generated, so a large draft of the buoy is needed to generate an greater buoyancy for diving TOSS (Figure S1). Combining with Figure 2a, it is not difficult to find $\Delta X_{I}<$ $\triangle X_{2}$.

\section{Supplementary Note2 :}

Based on the corresponding principle of sliding model TENG, assuming the effective contact area of TOSS is $\Delta S$, the surface charges density of PTFE is $\sigma$, the diameter of PTFE is $D$, the sliding distance is $\Delta h$, respectively. Meanwhile, based on the Figure 2a, we can find that the sliding distance of TOSS $(\Delta h)$ is basically equal to the wave-height $(H)$. According to the relevant theory and geometric relationship, these parameters have the close relationship with the transferred charges $(\Delta Q)$ of TOSS as follow:

$$
\begin{array}{r}
\Delta Q=\sigma \Delta S \\
\Delta Q=\pi \sigma D \Delta h=\pi \sigma D H
\end{array}
$$

In addition, according to the past work research, the open-circuit voltage $(V)$ of TOSS is proportional to the transferred charges $(\Delta Q)$, and the relevant of scale factor is $k$. Then, the following relational can be obtained.

$$
V=k \Delta Q=\pi k \sigma d \Delta h=\pi k \sigma D H
$$

Therefore, the $V$ and $\Delta Q$ can be applied to monitor the sliding distance $\Delta h$, and then achieve the detection of wave height $(H)$. 


\section{Supplementary Note 3 :}

To obtain the excellent performance of TOSS, good contact between the dielectric material and electrodes must always be maintained in the progress of sensor operation (Formula 3). Therefore, as is shown in Figure S4, assuming the internal diameter of external copper tube electrode is $D_{i}$, the outer diameter of internal tube electrode is $d_{o}$, and the thickness of dielectric material is $l$, there should be the following relation among three parameters:

$$
D i=d o+2 l
$$

\section{Supplementary Note4 :}

The motion mode of motor is depicted in Figure S6, which is reference to the previous research of ocean wave. The corresponding motion process is a reciprocating motion at a fixed acceleration (Figure 6a). The curve of motor speed and displacement over time are displayed in Figure 6b-c, which are combined with the many motion progress of formulas (Formula 5-6).

$$
\begin{gathered}
v_{i}=a t \\
\Delta h=1 / 2 a t^{2}
\end{gathered}
$$

Where $v_{i}$ is the speed of TOSS, $a$ is the acceleration of motor, $\Delta h$ is the displecement of TOSS and $t$ is the time.

Besides, according to the above conclusion and Note S1, it is easy to obtain that the relationship between current and velocity.

$$
\begin{gathered}
\Delta Q=\pi \sigma D v_{i}{ }^{2} / 2 a \\
V=\pi k \sigma D v_{i}{ }^{2} / 2 a \\
I=\Delta Q / t=\pi \sigma D \Delta h / t=\pi \sigma D v_{i} / 2
\end{gathered}
$$

Where $I$ is the short-circuit current.Therefore, it is clearly found that the obtained transferred charges and voltage spectra are similar with the displacement curve of 
motor, and the achieve current curve is similar with the speed of motor (Figure S7).

\section{Supplementary Note 5:}

In the table, the AL, DP, MV, TCV and ME represent the acceleration, displacement, measured value, theoretical calculation value and measurement error, respectively. Meanwhile, the theoretical calculation value of period is based on Figure S6, Note 2 and the basic physical formulas.

\section{Supplementary Note 6 :}

As shown in the Figure S9, it is easy to obtain the period information of waves by this ocean spectrum. Meanwhile, assuming the effective action length of waves on the buoy is $s$, and the action time of waves on the buoy is $\Delta t$. As a general rule, owing to the $s$ is a fixed value at the average state, we can obtain a spread speed and achieve the measurement of wave-velocity $(v)$ by adding a calibration factor analysis $(\alpha)$. The process is as follows:

$$
v=\alpha s / \Delta t
$$

Furthermore, according to the related definition of other ocean parameters, such as, wave-frequency $(f)$, wavelength $(L)$, wave-steepness $(\delta)$, and so on, which can also be painlessly calculated by the following formulas.

$$
\begin{gathered}
f=1 / T \\
L=v T \\
\delta=H / L
\end{gathered}
$$

\section{Supplementary Note 7 :}

More sensors can monitor more parameters, Figure S8 minutely describes the 
monitoring of wave propagation direction by using both of TOSS. In the picture, the two orange spheres stand for two TOSSs, the blue ray represents the spread direction of waves, and $S$ represents the distance between two TOSSs, and the included angle of between the connection of the two TOSSs and the wave propagation direction is expressed as $\theta$. For the value of $\mathrm{S}$ is known, the $\mathrm{v}$ can obtain from Note $\mathbf{3}$, and based on the time difference $(\Delta T)$ between two TOSSs for the same wave detection. According to the mathematical relationship between them (Formulas 10), which can achieve the value of $\theta$ and then obtain the propagation direction of waves.

$$
S \cdot \cos \theta=v \Delta T
$$

\section{Supplementary Note 8:}

Based on Figure S7-8 and Note 4, we can obtained the follow equation:

$$
\begin{gathered}
\Delta Q=\pi \sigma D v_{v}{ }^{2} / 2 a_{v} \\
V=\pi k \sigma D v_{v}{ }^{2} / 2 a_{v} \\
I=\pi \sigma D v_{v} / 2
\end{gathered}
$$

where $\Delta Q$ is the transferred charge of TOSS, $V$ is the open-circuit voltage, $I$ is the short-circuit current of TOSS, $k$ is the relevant scale factor of $V$ and wave-height $(H)$, $\sigma$ is the surface charges density of PTFE, $D$ is the diameter of PTFE, $v_{v}$ is the velocity of ocean-wave in the vertical direction and $a_{v}$ is the obtained acceleration of ocean-wave in the vertical direction. Compared with the real ocean-wave spectrum pattern and the vertical motion mode of ocean-wave simulated by motor, we can find that they are basically similar, so we can think that $a_{v}$ is basically a fixed constant. In addition, the projection area of the surface contacted by hollow buoy and seawater on the horizontal plane is fixed, we can use a circle with a radius of $R$ to represent it. Meanwhile, we can use the following formula 18 to easily transform the spectrum of wave-height for time $(H(t))$ into the shape spectrum of ocean-wave $((H(s))$.

$$
H(s)=v H(t)
$$

Where $v$ is the wave-velocity by TOSS. Therefore, based on the formulas $\mathbf{2 , 3}, \mathbf{1 5}, \mathbf{1 6}$ and the transferred charges or open-circuit voltage spectrum of TOSS from 
ocean-wave, we can get the following equations to obtain the kinetic energy spectrum of ocean-wave in the vertical direction by the following equals ( $W$ is the transverse width of ocean-wave) .

$$
\begin{aligned}
E_{k v}(t)= & m_{w}(s) \quad\left\{v_{v}(t)\right\}^{2} W / 4 R \\
& =\left\{\pi \rho_{s} R^{2} H(s)\right\}\left\{2 a_{v} \Delta Q(t) / \pi \sigma D\right\} W / 4 R \\
& =a_{v} \rho_{s} v R W\{\Delta Q(t)\}^{2} / 2 \pi \sigma^{2} D^{2} \\
E_{k v}(t) & =m_{w}(s)\left\{v_{v}(t)\right\}^{2} W / 4 R \\
& =\left\{\pi \rho_{s} R^{2} H(s)\right\}\left\{2 a_{v} V(t) / k \pi \sigma D\right\} W / 4 R \\
& =a_{v} \rho_{s} v R W\{V(t)\}^{2} / 2 \pi k^{2} \sigma^{2} D^{2}
\end{aligned}
$$

Where $E_{k v}$ is the kinetic energy of ocean-wave spectrum in the vertical direction, $m_{w}$ is the mass of seawater on the TOSS, $\rho_{s}$ is the density of seawater, $\Delta Q(t)$ is the transferred charge spectrum of TOSS and $V(t)$ is the open-circuit voltage spectrum of TOSS.

\section{Supplementary Note 9:}

The kinetic energy of ocean-wave in the propagation direction can be regarded as the kinetic energy of the seawater forming the ocean-wave in this direction. Therefore, the kinetic energy spectrum of ocean-wave in the propagation direction can be achieved by formula 21 or 22 ( Figure S14).

$$
\begin{aligned}
& E_{k p}(t)=m_{w}(s) v^{2} W / 4 R=\pi \rho_{s} v^{2} R W H(s) / 4=\rho_{s} v^{3} R W \Delta Q(t) / 4 \sigma D \\
& E_{k p}(t)=m_{w}(s) v^{2} W / 4 R=\pi \rho_{s} v^{2} R W H(s) / 4=\rho_{s} v^{3} R W V(t) / 4 k \sigma D
\end{aligned}
$$

\section{Supplementary Note 10:}

As the seawater can be regarded as a substance with uniform density, the barycenter of ocean-wave in different position is the located at the center of ocean-wave in the vertical direction as shown in the Figure S15. Therefore, based on the Note 9, the 
gravitational potential energy spectrum of ocean-wave can be obtained by the following formula.

$$
\begin{gathered}
E_{k p}(t)=m_{w}(s) g W H(s) / 4 R=\rho_{s} g v^{2} R W\{\Delta Q(t)\}^{2} / 4 \pi \sigma D \\
E_{k p}(t)=m_{w}(s) g W H(s) / 4 R=\rho_{s} g v^{2} R W\{V(t)\}^{2} / 4 \pi k^{2} \sigma^{2} D^{2}
\end{gathered}
$$




\section{Supplementary Figures}

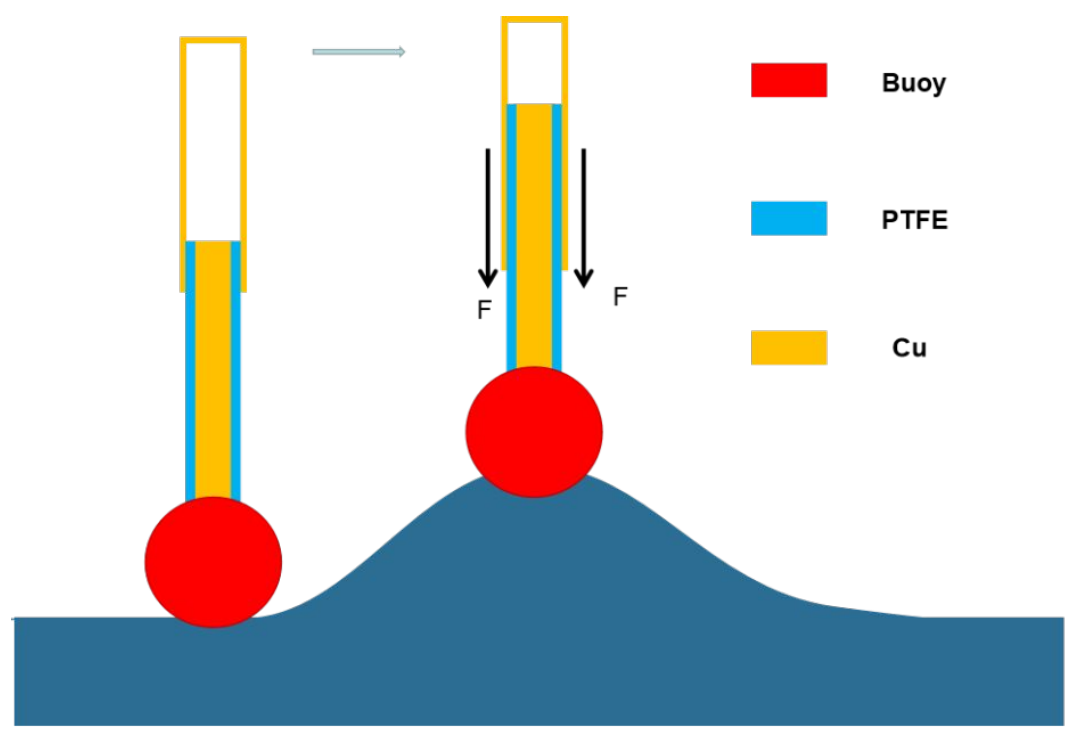

Supplementary Figure 1. The error analysis of wave height measurement by the TOSS.
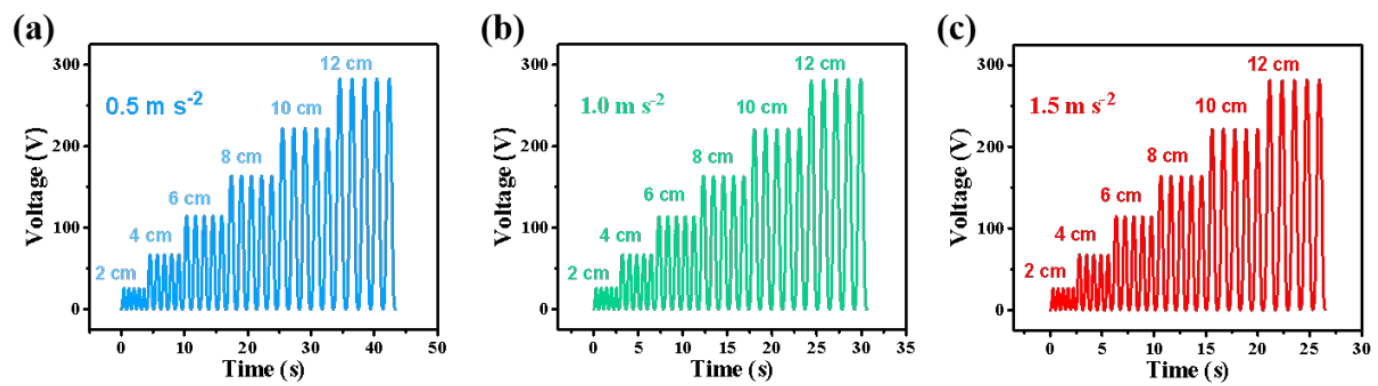

Supplementary Figure 2. The open-circuit voltage of TOSS at the different wave height and various of acceleration with (a) $0.5 \mathrm{~m} \mathrm{~s}^{-2}$, (b) $1.0 \mathrm{~m} \mathrm{~s}^{-2}$, and (c) $1.5 \mathrm{~m} \mathrm{~s}^{-2}$. 
(a)

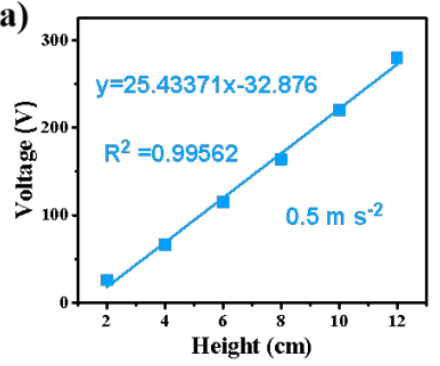

(b)

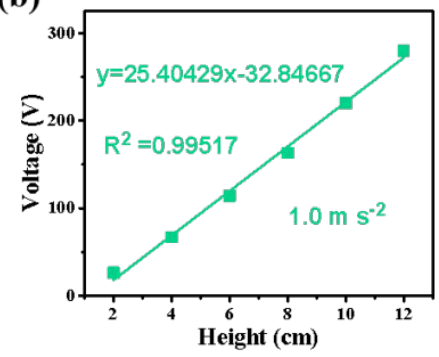

(c)

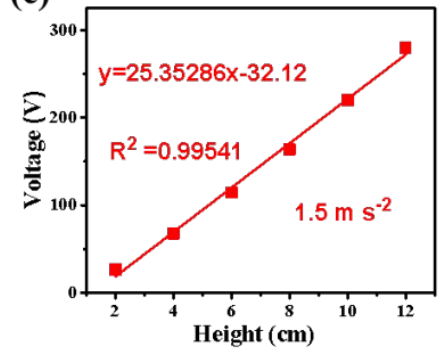

Supplementary Figure 3. The linear relationship between wave-height and the open-circuit voltage signals of TOSS under the different acceleration with (a) $0.5 \mathrm{~m}$ $\mathrm{s}^{-2}$, (b) $1.0 \mathrm{~m} \mathrm{~s}^{-2}$, and (c) $1.5 \mathrm{~m} \mathrm{~s}^{-2}$.

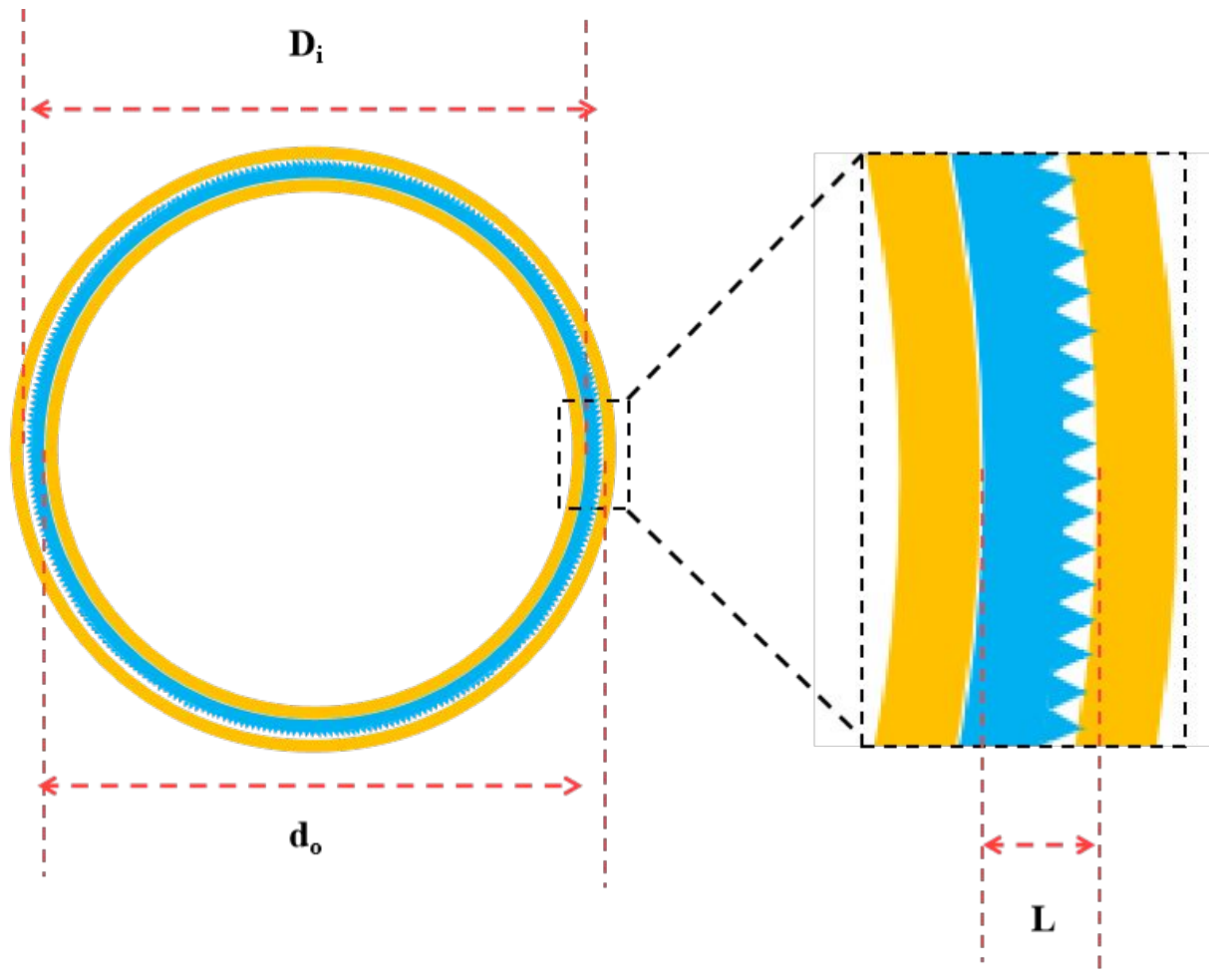

Supplementary Figure 4. The Geometric design of TOSS. 


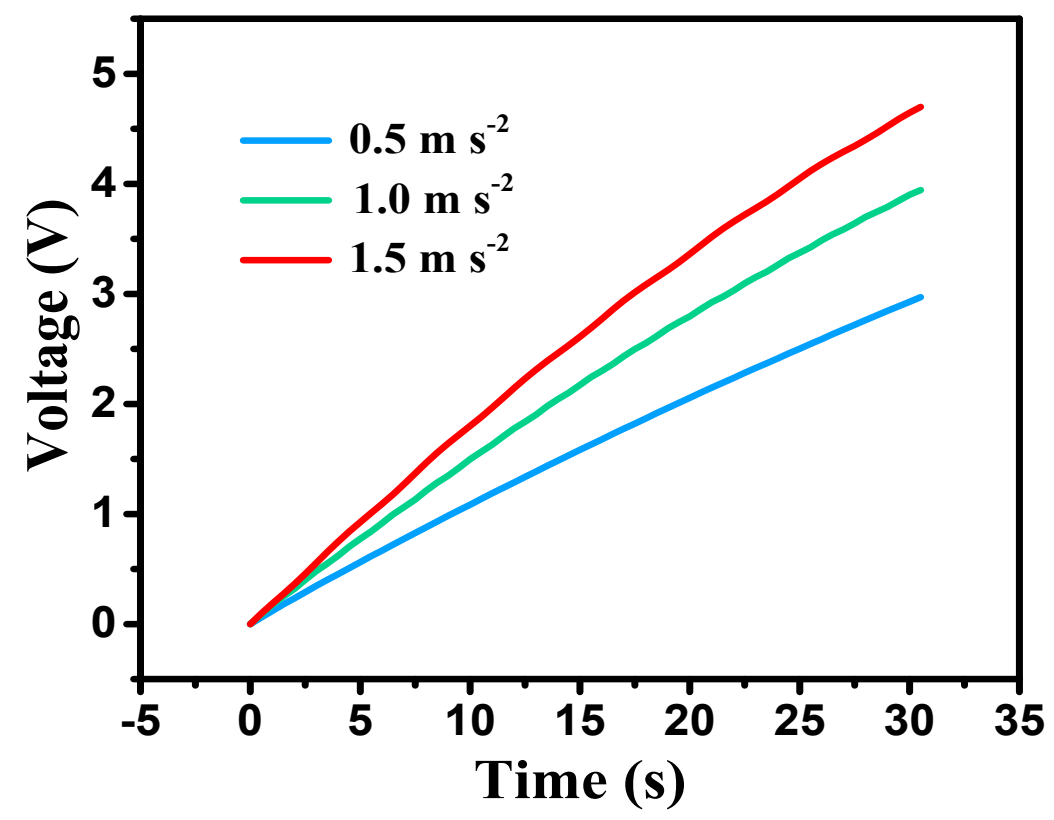

Supplementary Figure 5. The charging-capacitor curve of TOSS under different acceleration.

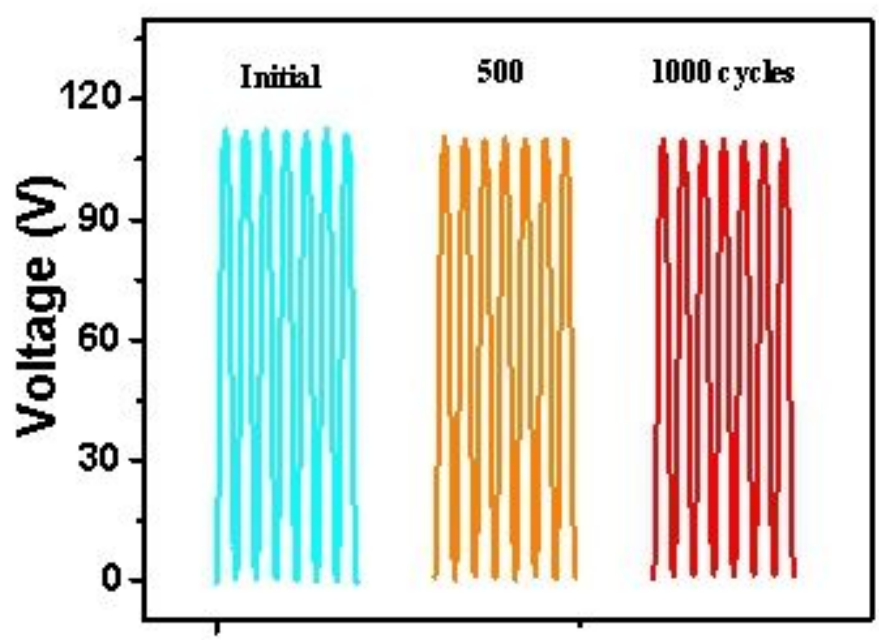

Supplementary Figure 6. A test of the stability of TOSS by linear motor. 
(a)

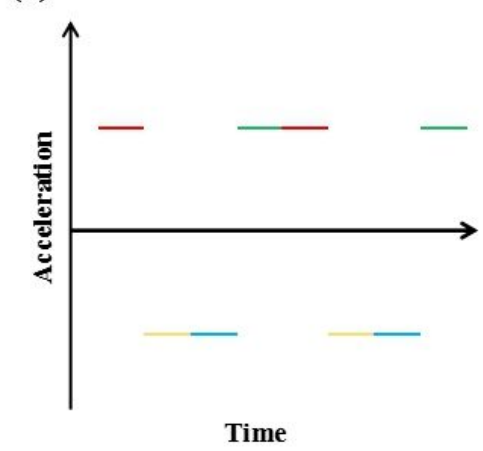

(b)

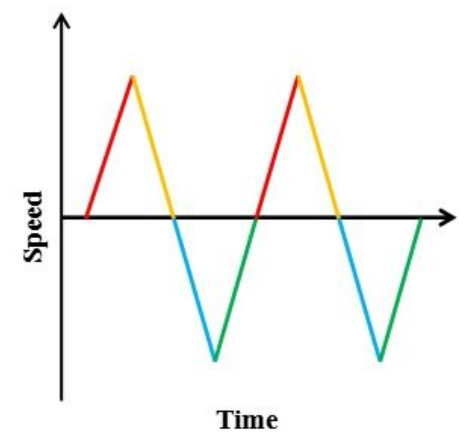

(c)

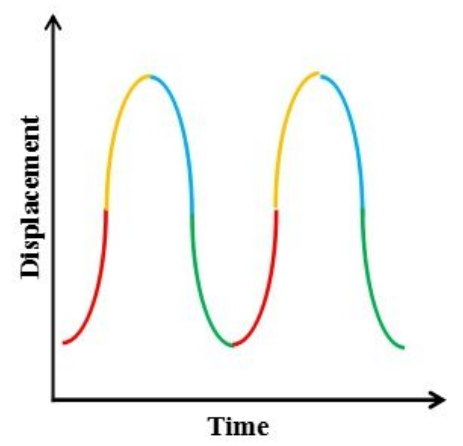

Supplementary Figure 7. The physical motion mode of vertical linear motor. (a) Acceleration change of the motor. (b) Speed change of the motor. (c) Displacement change of motor.

(a)

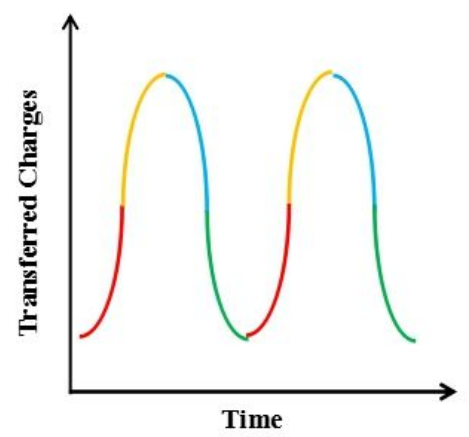

(b)

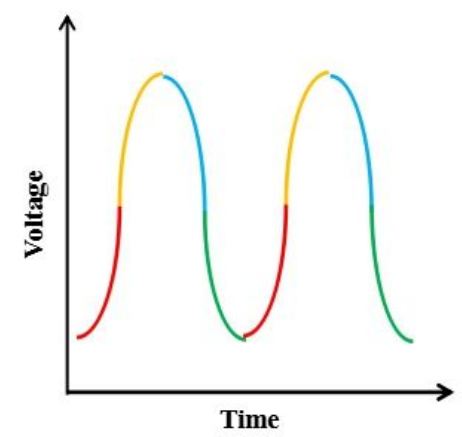

(c)

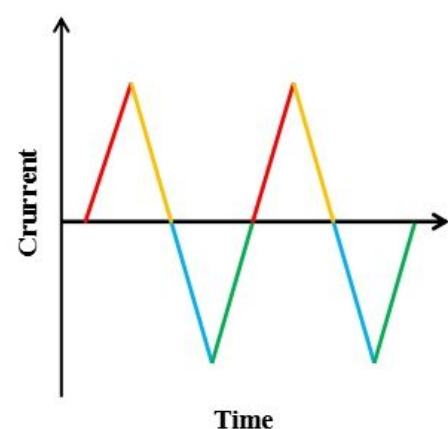

Supplementary Figure 8. The ideal signal spectra of TOSS. (a) The transferred charges spectrum of TOSS. (b) The open-circuit voltage spectrum of TOSS. (c) The short-circuit current spectrum of TOSS. 
(a)

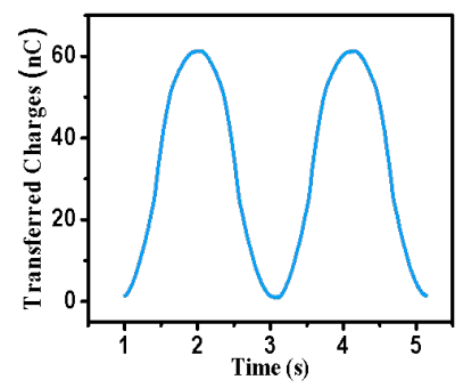

(b)

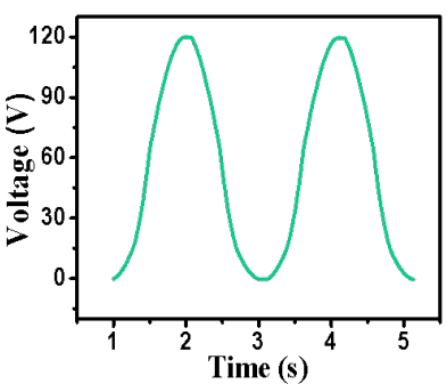

(c)

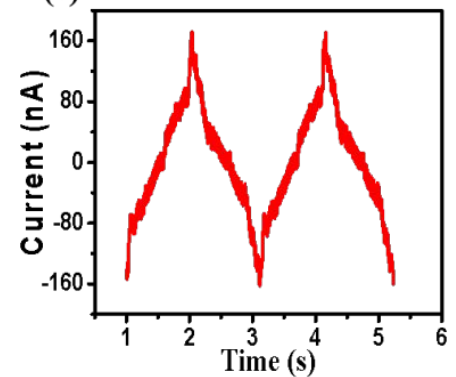

Supplementary Figure 9. The real obtain spectra by TOSS. (a) The transferred charges spectrum, (b) open-circuit voltage spectrum, (c) short-circuit current spectrum by TOSS.

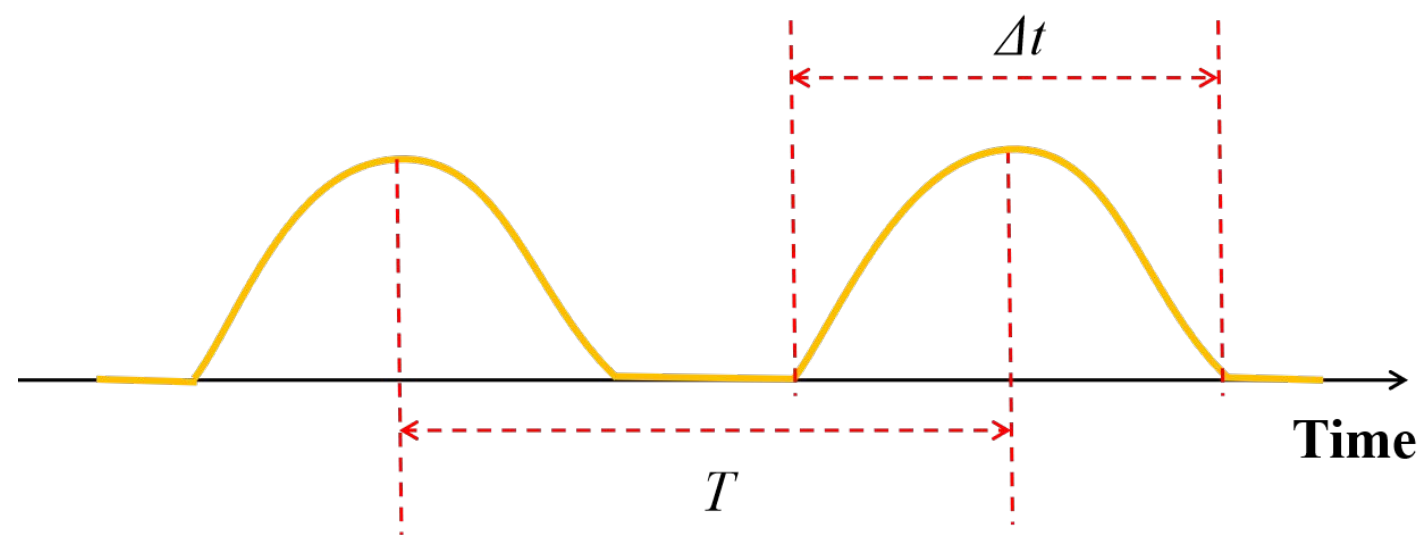

Supplementary Figure 10. The analysis of ocean spectral structure obtained by the TOSS. 


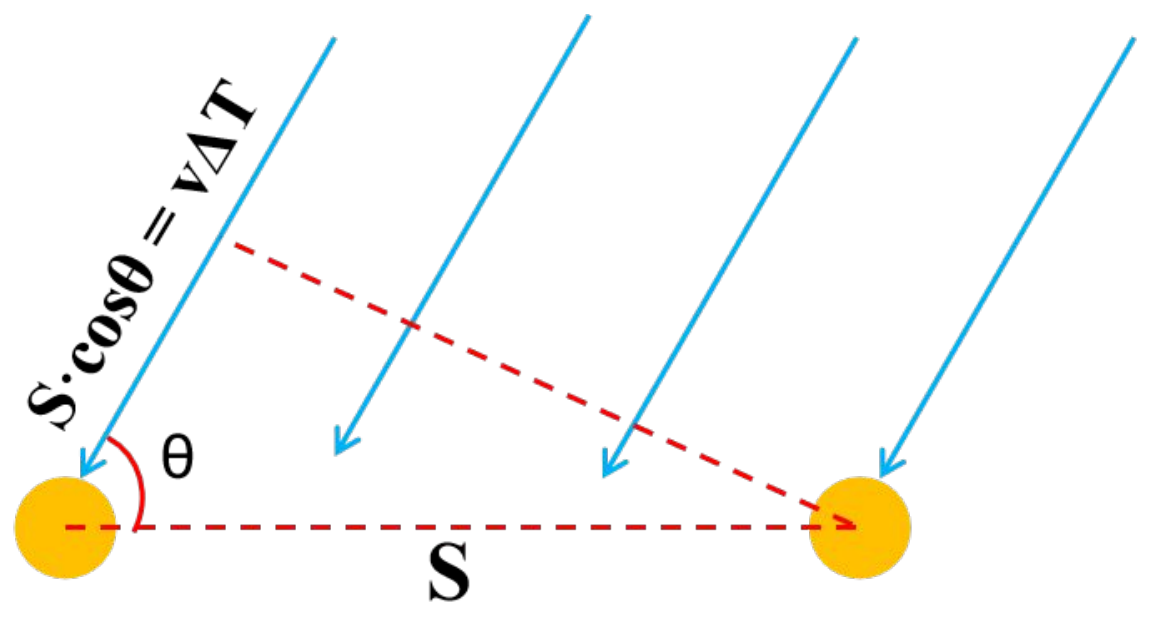

Supplementary Figure 11. The principle of detecting the direction of wave propagation by TOSS.

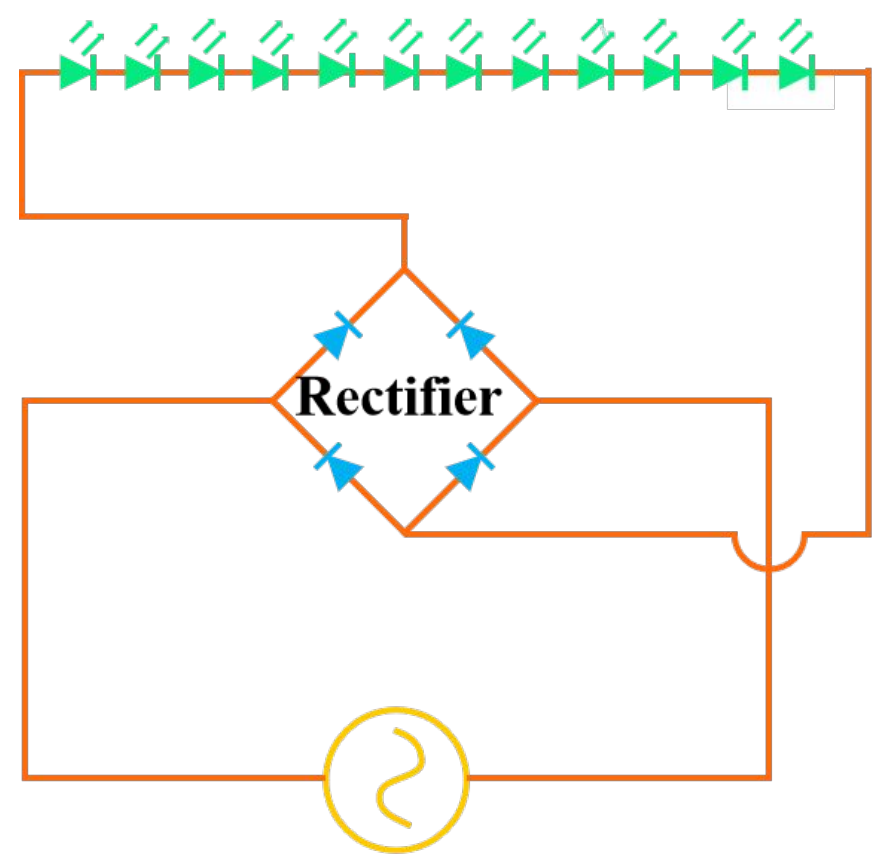

OSS-TENG

Supplementary Figure 12. The corresponding circuit diagram of lighting LEDs. 


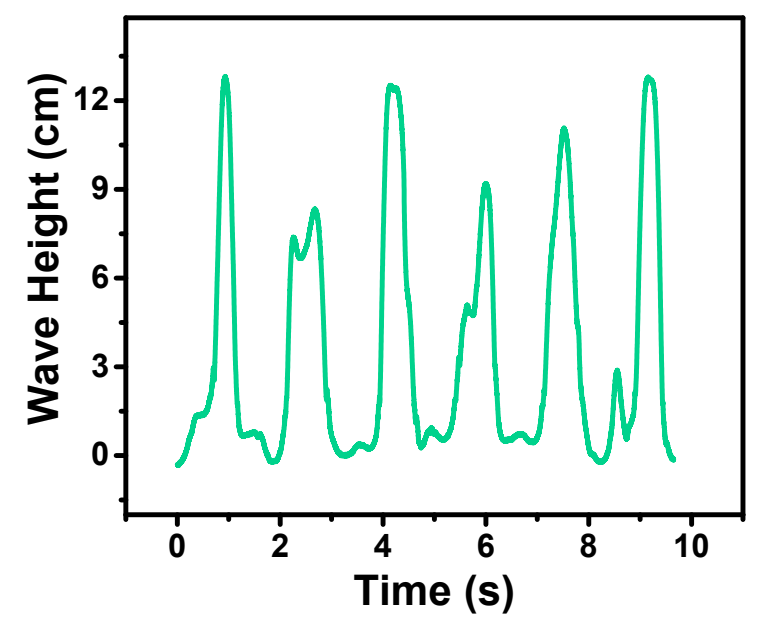

Supplementary Figure 13. The water wave height spectrum extracting from open-circuit voltage by using TOSS.

(a)

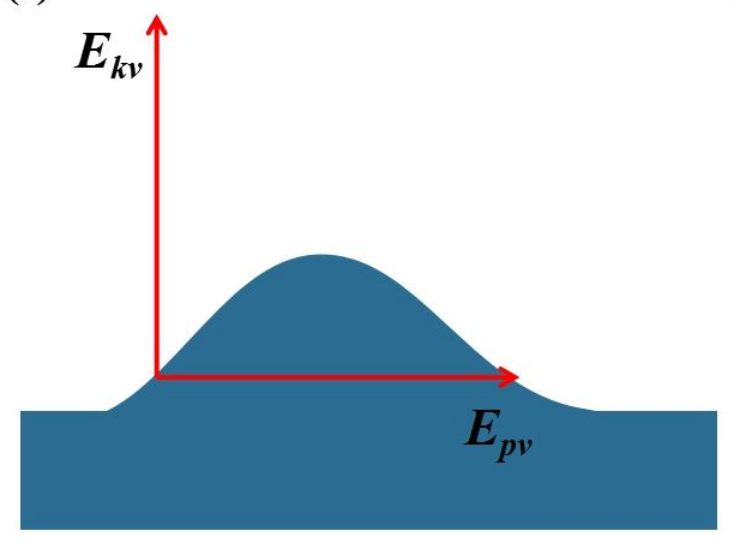

(b)

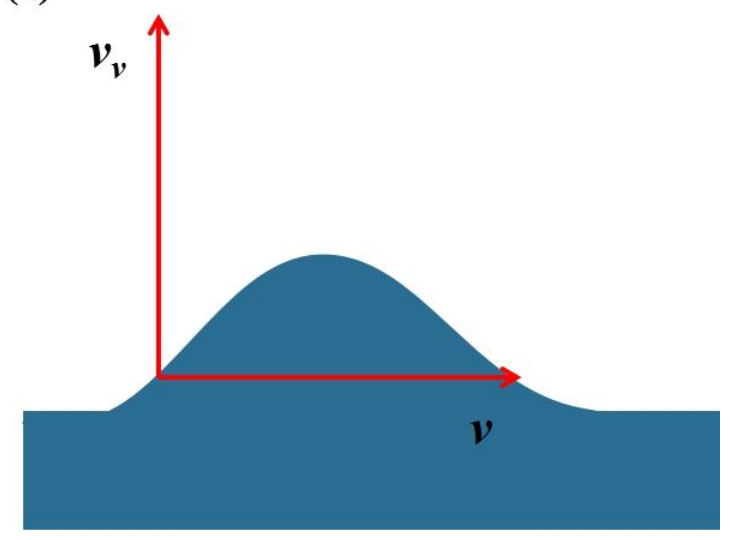

Supplementary Figure 14. The decomposition diagram of kinetic energy and velocity of ocean-wave. 


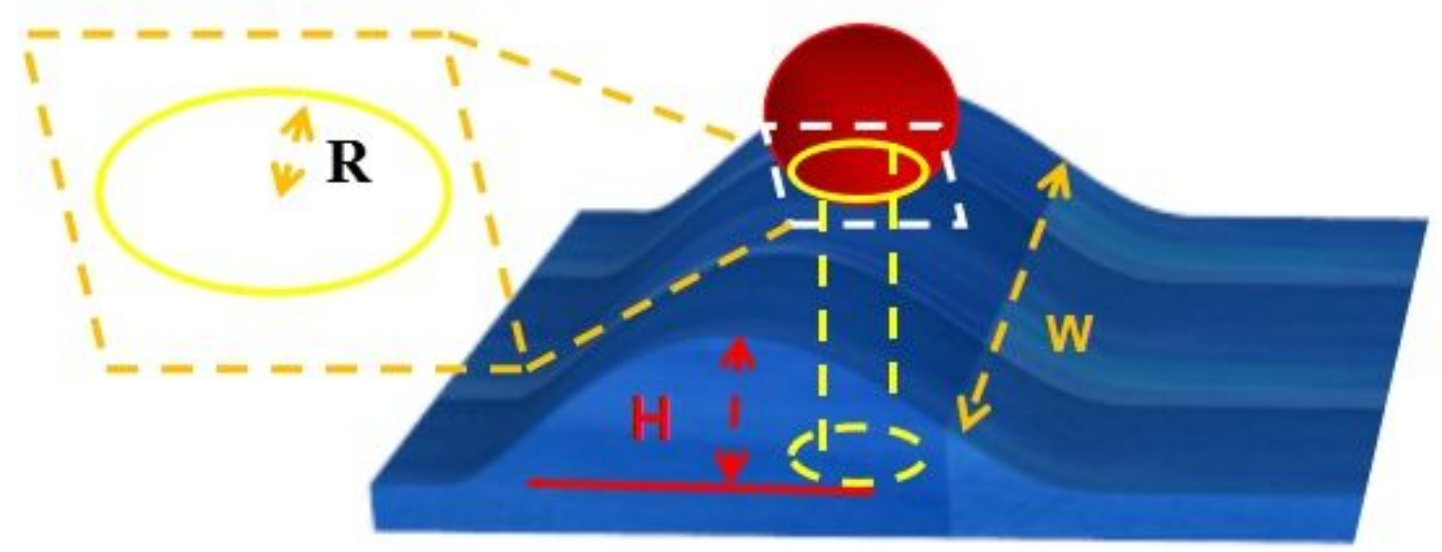

Supplementary Figure 15. The structural analysis diagram of the contact between the hollow buoy and ocean-wave.

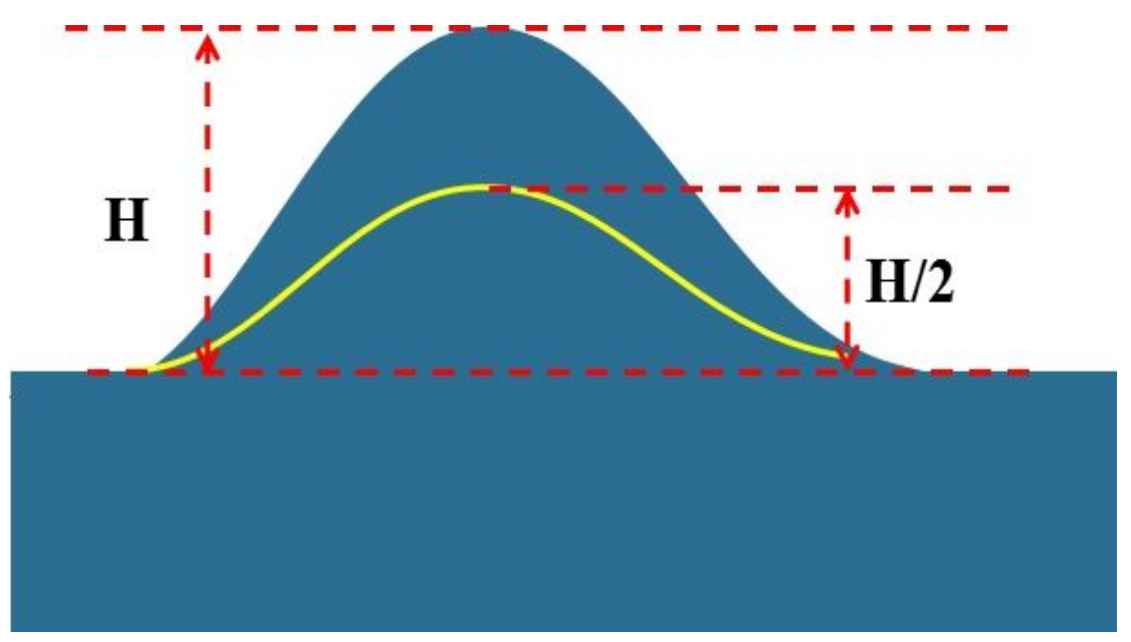

Supplementary Figure 16. The barycenter distribution of ocean-wave in the different position. 


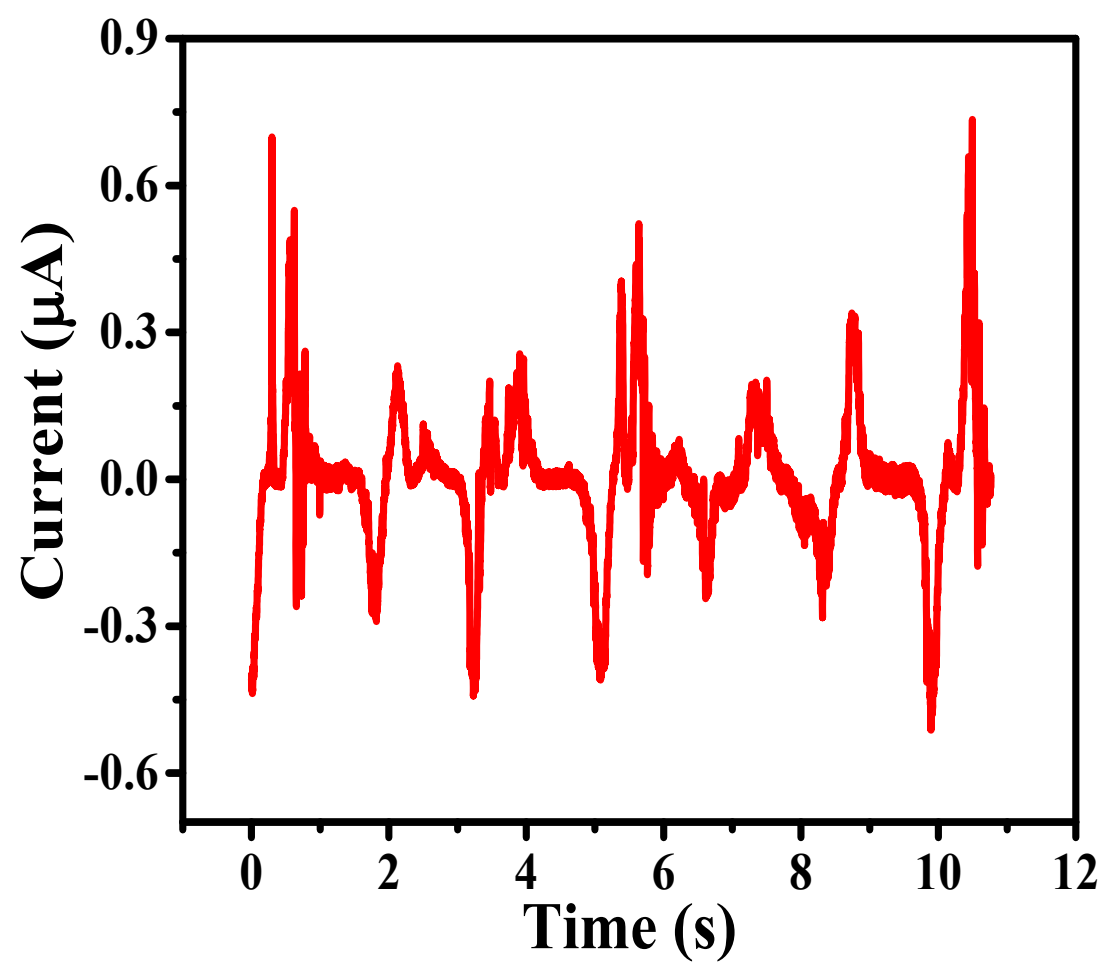

Supplementary Figure 17. The short-circuit current spectrum of TOSS by driving the simulative water waves. 


\section{Supplementary Tables}

Supplementary Table 1. Error analysis of wave period extracted by the transferred charges of TOSS.

Error Analysis Table for Wave Period Testing （Transferred Charges Testing)

\begin{tabular}{|c|c|c|c|c|c|c|c|c|c|}
\hline $\mathrm{AL}$ & & $0.5 \mathrm{~m}$ & $\mathrm{~s}^{-2}$ & & & $0 \mathrm{~m} \mathrm{~s}^{-2}$ & & 1.5 & $\mathrm{~m} \mathrm{~s}^{-2}$ \\
\hline DP & MV (ms) & $\mathrm{CV}$ (ms) & ME & MV (ms) & TVC $(\mathrm{ms})$ & ME & MV (ms) & TVC (ms) & ME \\
\hline 2 & 4794 & 4800 & $0.125 \%$ & 4565 & 4566 & $-0.015 \%$ & 4462 & 4462 & $0.003 \%$ \\
\hline 4 & 5131 & 5131 & $0.007 \%$ & 4801 & 4800 & $0.021 \%$ & 4652 & 4653 & $-0.026 \%$ \\
\hline 6 & 5383 & 5386 & $0.049 \%$ & 4980 & 4980 & $0.004 \%$ & 4800 & 4800 & $0.000 \%$ \\
\hline 8 & 5597 & 5600 & $0.054 \%$ & 5132 & 5131 & $0.012 \%$ & 4928 & 4924 & $0.086 \%$ \\
\hline 10 & 5791 & 5789 & $-0.037 \%$ & 5266 & 5265 & $0.021 \%$ & 5035 & 5033 & $0.044 \%$ \\
\hline 12 & 5965 & 5960 & $-0.091 \%$ & 5387 & 5386 & $0.025 \%$ & 5140 & 5131 & $0.168 \%$ \\
\hline
\end{tabular}

Supplementary Table 2. Error analysis of wave period extracted by the open-circuit voltage of TOSS.

\begin{tabular}{|c|c|c|c|c|c|c|c|c|c|}
\hline \multicolumn{6}{|c|}{ Error Analysis Table for Wave Period Testing } & \multicolumn{4}{|c|}{ (Open-Circuit Voltage Testing) } \\
\hline \multirow{2}{*}{$\begin{array}{l}\mathrm{AL} \\
\mathrm{DP}\end{array}$} & \multicolumn{3}{|c|}{$0.5 \mathrm{~m} \mathrm{~s}^{-2}$} & \multicolumn{4}{|c|}{$1.0 \mathrm{~m} \mathrm{~s}^{-2}$} & \multicolumn{2}{|c|}{$1.5 \mathrm{~m} \mathrm{~s}^{-2}$} \\
\hline & MV (ms & $\mathrm{CV}$ (ms) & $\mathrm{ME}$ & $\mathrm{MV}$ (ms) & TVC (ms) & $\mathrm{ME}$ & $\mathrm{MV}$ (ms) & $\mathrm{TVC}(\mathrm{ms})$ & $\mathrm{ME}$ \\
\hline 2 & 4797 & 4800 & $-0.063 \%$ & 4564 & 4566 & $-0.037 \%$ & 4462 & 4462 & $0.003 \%$ \\
\hline 4 & 5130 & 5131 & $-0.027 \%$ & 4799 & 4800 & $-0.021 \%$ & 4653 & 4653 & $-0.004 \%$ \\
\hline 6 & 5385 & 5386 & $-0.012 \%$ & 4980 & 4980 & $0.004 \%$ & 4800 & 4800 & $0.000 \%$ \\
\hline 8 & 5598 & 5600 & $-0.036 \%$ & 5131 & 5131 & $-0.007 \%$ & 4923 & 4924 & $-0.015 \%$ \\
\hline 10 & 5786 & 5789 & $-0.049 \%$ & 5265 & 5265 & $0.002 \%$ & 5033 & 5033 & $0.004 \%$ \\
\hline 12 & 5960 & 5960 & $0.007 \%$ & 5386 & 5386 & $0.007 \%$ & 5132 & 5131 & $0.012 \%$ \\
\hline
\end{tabular}

